\title{
SERIES NO. 1 \\ IDENTIFICATION AND HPLC QUANTIFICATION OF CAROTENOIDS OF THE FRUIT PULP OF CHRYSOPHYLLUM ROXBURGHII
}

\author{
U. G. CHANDRIKA ${ }^{1}$, E. R. JANSZ ${ }^{1 *}$ and N. D. WARNASURIYA ${ }^{2}$ \\ 1 Department of Biochemistry, Faculty of Medical Sciences, University of Sri Jayewardenepura, Nugegoda. \\ 2 Department of Paediatrics, Faculty of Medical Sciences, University of Sri Jayewardenepura, Nugegoda.
}

(Accepted: 21 December 2004)

\begin{abstract}
Carotenoids of the fruit pulp of Chrysophyllum roxburghii (Sinhala: lavalu) amounted to about $180 \mathrm{mgkg}^{-1}$ by fresh weight. The carotenoids were isolated by open column chromatography (MgO:Celite 1:1) using mixtures of petroleum ether $40-60^{\circ} \mathrm{C}$ and acetone and identified by UV/visible spectra, chemical tests, and High Performance Liquid Chromatography (HPLC) using authentic standards and a photodiode array detector (PAD). The major carotenoid was trans-violaxanthin $\left(113 \mathrm{mgkg}^{-1}\right)$. Also present was cisviolaxanthin, neoxanthin, $\beta$-cryptoxanthin monoepoxide, lutein, $\beta$-cryptoxanthin, $\zeta$-carotene and $\beta$-carotene. The retinol equivalent of the pulp was only $68 \mathrm{RE} / 100 \mathrm{~g}$. The study shows that Chrysophyllum roxburghii is not a good source of provitamin A. Further as violaxanthin is reported to be not absorbed by humans, it is of no use as a dietary antioxidant. However, as trans-violaxanthin can be obtained in quantity in the pure crystalline state, directly from the column and has the benefit of eluting at $20 \%$ acetone away from most carotenoids, the compound will be useful as a standard for HPLC analysis of carotenoids from other fruits and leaves.
\end{abstract}

Key words: carotenoids, Chrysophyllum roxburghii, Lavalu, violaxanthin.

\section{INTRODUCTION}

Chrysophyllum roxburghii (Indian star apple) and Chrysophyllum monopyrenum (wild star apple) both known as Lavalu (Sinhala) are the two species of Chrysophyllum found in the Indian sub continent. ${ }^{1}$ C. lanceolatum is a synonym for $C$. roxburghii.

No studies have been reported in published, refereed journals on the carotenoids of $C$. roxburghii fruit. Howerver, a thesis ${ }^{2}$ states that the fruit has at least 18 carotenoids and a total carotenoid content of $112 \mathrm{mgkg}^{-1}$ (as $\beta$ carotene). The carotenoids reported are phytoene, phytofluene, $\alpha$-carotene, $\beta$-carotene, $\zeta$-carotene, pro- $\gamma$-carotene, 5,6 epoxydihydro $\beta$-carotene, 5,6 , $5 ', 6$ ' epoxy diepoxy tetrahydro $\beta$-carotene, $\beta$ cryptoxanthin, microxanthin, zeaxanthin, and 3 - hydroxy sintaxanthin. Some of these are not listed in a recent compendium on carotenoids naturally occurring in plants. ${ }^{3}$ Further, the experimental techniques and identification procedures used in the above study are not in line with modern analysis procedures. There is, therefore, a possibility that some of these are artifacts of isolation.

The present study deals with the characterization of the carotenoids of Sri Lankan lavalu (Chrysophyllum roxburghii G. Don) as part of our overall research program to study the carotenoid composition of Sri Lankan yellow fruits and leafy vegetables. It is especially important in Sri Lanka and other developing countries to identify as many yellow fruits to determine whether they can be used as a pro-vitamin A source or antioxidant. The objective of this study was to determine the carotenoid profile of lavalu using chromatography, chemical tests, UV spectrometry and High Performance Liquid Chromatography (HPLC) with authentic standards. HPLC with UV-visible photodiode array (PDA) detector was also used for the identification and quantification.

\section{METHODS AND MATERIALS}

Isolation of standards by Open Column Chromatography (OCC): Parsley (Petroselinum crispum L.) was used as the source of standards (neoxanthin, violaxanthin, lutein and $\beta$-carotene) because it is easy to extract and has high carotenoid content. To obtain the standards, the carotenoids were extracted with cold acetone, partitioned to petroleum ether, concentrated in the rotary evaporator and separated in an open column of $\mathrm{MgO}$ (Maerk Germany): Celite (1:1 activated for $2 \mathrm{~h}$ at $\left.110^{\circ} \mathrm{C}\right) .{ }^{3,4}$ 
Preparation of column: A chromatographic glass tube was mounted on a suction flask. A small glass wool plug was placed at the bottom of the chromatographic tube. The separating medium (MgO: Celite 1:1) was added loosely up to a height of $20 \mathrm{~cm}$ and continuous moderate vacuum was applied from a water aspirator. A flat instrument was used to press down the absorbent and flatten the surface. The column was topped with a $1 \mathrm{~cm}$ layer of anhydrous sodium sulphate to ensure that no residual water gets into the adsorbent. The column was pre-equilibrated with petroleum ether (boiling range $40-60^{\circ} \mathrm{C}$ ).

Developing the column: The carotenoid solution was carefully poured into the column and the sample layer was allowed to reach almost to the surface of the sodium sulphate layer. The column was eluted with $100 \%$ petroleum ether, gradient of 1\%-8\% diethyl ether in petroleum ether and 1$50 \%$ acetone in petroleum ether.

An aliquot was taken from each isolate to verify purity by HPLC. All aliquots were dried under $\mathrm{N}_{2}$ and immediately before injection, dissolved in $1 \mathrm{ml}$ HPLC grade acetone, filtered through a $0.22 \mu \mathrm{m}$ polyethylene teflon (PTEF) syringe filter (Millipore) directly to the sample vials and $10 \mu \mathrm{l}$ injected into the HPLC. Once the desired purity was confirmed using HPLC, the concentration of the pure standards were determined spectrophometrically, using the following $\mathrm{A}_{\mathrm{cm}}$ values: $\beta$-carotene, 2592 in petroleum ether; lutein, 2550 in ethanol; violaxanthin, 2550 in ethanol: neoxanthin, 2443 in ethanol. Aliquots of the standard mixtures were quantitatively transferred into screw-capped culture tube, dried under nitrogen and stored in freezer until use.

\section{Calculation of carotenoid concentration:}

$$
X(\mu \mathrm{g})=\frac{\mathrm{A} \times \mathrm{Y}(\mathrm{ml}) \times 10^{6} \times \text { purity }}{\mathrm{A}_{\mathrm{cm}}^{1 \%} \times 100}
$$

$\mathrm{X}$ is weight $(\mu \mathrm{g})$ of the carotenoid standard, $\mathrm{Y}$ is the volume of the solution that gives an absorbance of $A$ at specified wavelength, $\mathrm{A}^{1 \%}$ is the absorption coefficient of the carotenoid in the solvent used.
$A_{x}=$ peak area of the carotenoid

$\mathrm{C}_{\mathrm{s}}=$ concentration of the standard

$\mathrm{A}_{\mathrm{s}}=$ peak area of the standard

Preparation of standard solution and construction of the standard curves for qualitative analysis of carotenoids from lavalu (violaxanthin, lutein, neoxanthin and $\beta$-carotene):

HPLC chromatogrms of the carotenoid standards are shown in Figure 1.

Construction of standard curve: Aliquots of the carotenoid isolates (in petroleum ether) were taken in volumes which gave the relative proportion found in the sample, mixed, concentrated, made up to $50 \mathrm{ml}$. For the standard curves, duplicate aliquots of $1,2,3,4$, and $5 \mathrm{ml}$ were transferred to culture tubes, dried under $\mathrm{N}_{2}$, and just before injection, dissolved in $1 \mathrm{ml}$ of acetone and filtered through a $0.22 \mu \mathrm{m}$ PTFE syringe filter (Millipore); $10 \mu \mathrm{l}$ was injected into the HPLC equipment.

Identification and quantification of the carotenoids of lavalu (Chrysophyllum Roxburghii G. Don):

Qualitative analysis: Ripe fruits of lavalu were selected from Piliyandala and Kadawata areas in Sri Lanka. The fruits were hard, oval shaped with a small point at the apex. The diameter of the fruits varied from $5-13 \mathrm{~cm}$ with $4-8$ seeds arranged steliately inside the flesh. Fruits were quartered and seeds and peel removed from two opposite sections of each fruits and the pulp homogenized in a blender. Fruit carotenoids are complex, qualitatively different and esterified. Analysis was carried out according to RodriguezAmaya (1999). ${ }^{3}$ The procedure involved the following. Homogenized fruit pulp (125 g) containing carotenoids was extracted with cold acetone, partitioned to petroleum ether, saponified with an equal volume of $10 \% \mathrm{KOH}$ in methanol at room temperature overnight, washed, concentrated, dried with $\mathrm{Na}_{2} \mathrm{SO}_{4}$ and separated on a $\mathrm{MgO}$ :celite as described above. Identification of carotenoids were carried out by the methods of Rodriguez-Amaya (1999). ${ }^{30}$ This study involved the combined use of the retention times, co- 

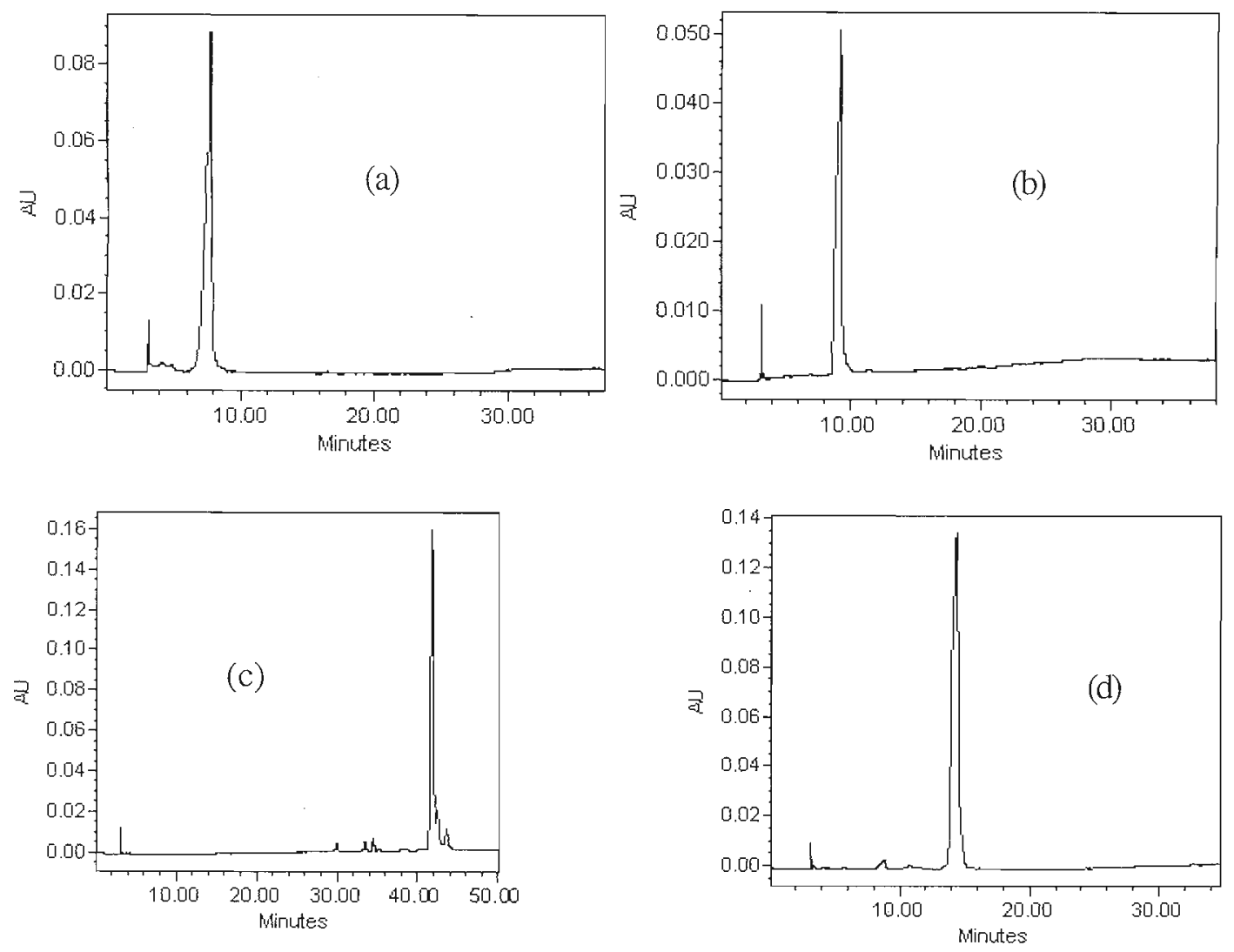

Figure 1: HPLC chromatograms of the carotenoid standards from Parsley (a) neoxanthin (b) violaxanthin (c) $\beta$-carotene (d) lutein

chromatography with authentic samples of neoxanthin, violaxanthin, lutein and $\beta$-carotene, the visible absorption spectra, and for xanthophylls, chemical tests such as acetylation with acetic anhydride of secondary hydroxy groups (as in lutien, violaxanthin, neoxanthin), methylation with acidic methanol of allylic secondary hydroxyl groups (as in lutein), and epoxide-furanoid rearrangement of 5,6, epoxy groups (as in violaxanthin and neoxanthin)

Quantitative analysis: To determine the mean carotenoid composition four batches of samples were analysed in duplicate. For each sample, fruit were quartered, seeds and peel were removed from two opposite sections of each fruit and the pulp was homogenized in a blender. Approximately $5 \mathrm{~g}$ (weighed accurately) were used for extraction. Extraction and saponification were carried out as described under qualitative analysis. Then $5.0 \mathrm{ml}$ of sample was evaporated to dryness immediately before injection to the HPLC, dissolved in a solution of acetone after filtering using a syringe filter.
HPLC conditions: The HPLC analysis was performed on Waters separation module (model 2690) equipped with quaternary pump, four channel in-line vacuum degasser, and auto sampler injector, controlled by Millenium 2010 workstation using a monomeric $\mathrm{C}_{18}$ column (Waters Spherisorb $\mathrm{S}_{3}$ ODS2), $3 \mu \mathrm{m}, 4.6 \times 150 \mathrm{~mm}$. The mobile phase consisted of acetonitirile, methanol, and ethyl acetate containing $0.05 \%$ of TEA (triethylamine) used at a flow rate of $0.5 \mathrm{ml} /$ min. A gradient was applied from 95:5:0 to 60:20:20 in $20 \mathrm{~min}$, maintaining this proportion until the end of the run. A UV-visible photodiode array detector (Waters model 996) with a millennium 32 software was used.

Chemical tests: Chemical tests were conducted to verify the type and position of substituents in xanthophylls and iodine-catalyzed isomerization reaction was carried out to verify the geometric configuration as follows $\mathrm{s}^{3}$ :

Acetylation of primary and secondary hydroxyl groups: The carotenoid (about $0.1 \mathrm{mg}$ ) was 
dissolved in $2 \mathrm{ml}$ pyridine and $0.2 \mathrm{ml}$ acetic anhydride was added. The reaction mixture was left in the dark at room temperature for $21 \mathrm{~h}$. The carotenoid was then transferred to petroleum ether, concentrated, and applied on a silica thin layer plate. The developing solvent was $5 \%$ methanol in toluene. The number of hydroxy groups were determined by the number of acetyl derivative TLC spots as the derivatisation agent was limiting.

Methylation of allylic hydroxyl groups: The carotenoid was dissolved in $5 \mathrm{ml}$ methanol and a few drops of $0.2 \mathrm{~N}$ hydrochloric acid was added. The reaction was allowed to proceed at room temperature in the dark for $3 \mathrm{~h}$. The carotenoids were then transferred to petroleum ether and subjected to TLC as described above.

Epoxide-furanoid rearrangement: The carotenoids were dissolved in ethanol and UV/visible spectrum
(300-600 nm) was recorded. A few drops of $0.1 \mathrm{~N}$ hydrochloric acid was added. The spectrum was again recorded after $3 \mathrm{~min}$. For each epoxide group a twenty nanometer hypsochromic shift was manifested.

Iodine-catalized cis-trans isomerization: A few crystals of iodine were dissolved in petroleum ether. The carotenoid spectrum was recorded in petroleum ether and a drop of the iodine solution was added. The spectrum was taken after 3 min exposure to light. The $\lambda_{\max }$ of trans will shift $3-5$ $\mathrm{nm}$ to a lower wavelength and of cis to a $3-5 \mathrm{~nm}$ higher wavelength.

\section{RESULTS AND DISCUSSION}

Figure 2 shows a HPLC chromatogram of the lavalu carotenoids and Table 1 represents identification. The main carotenoid in lavalu was identified as violaxanthin. Quantification of
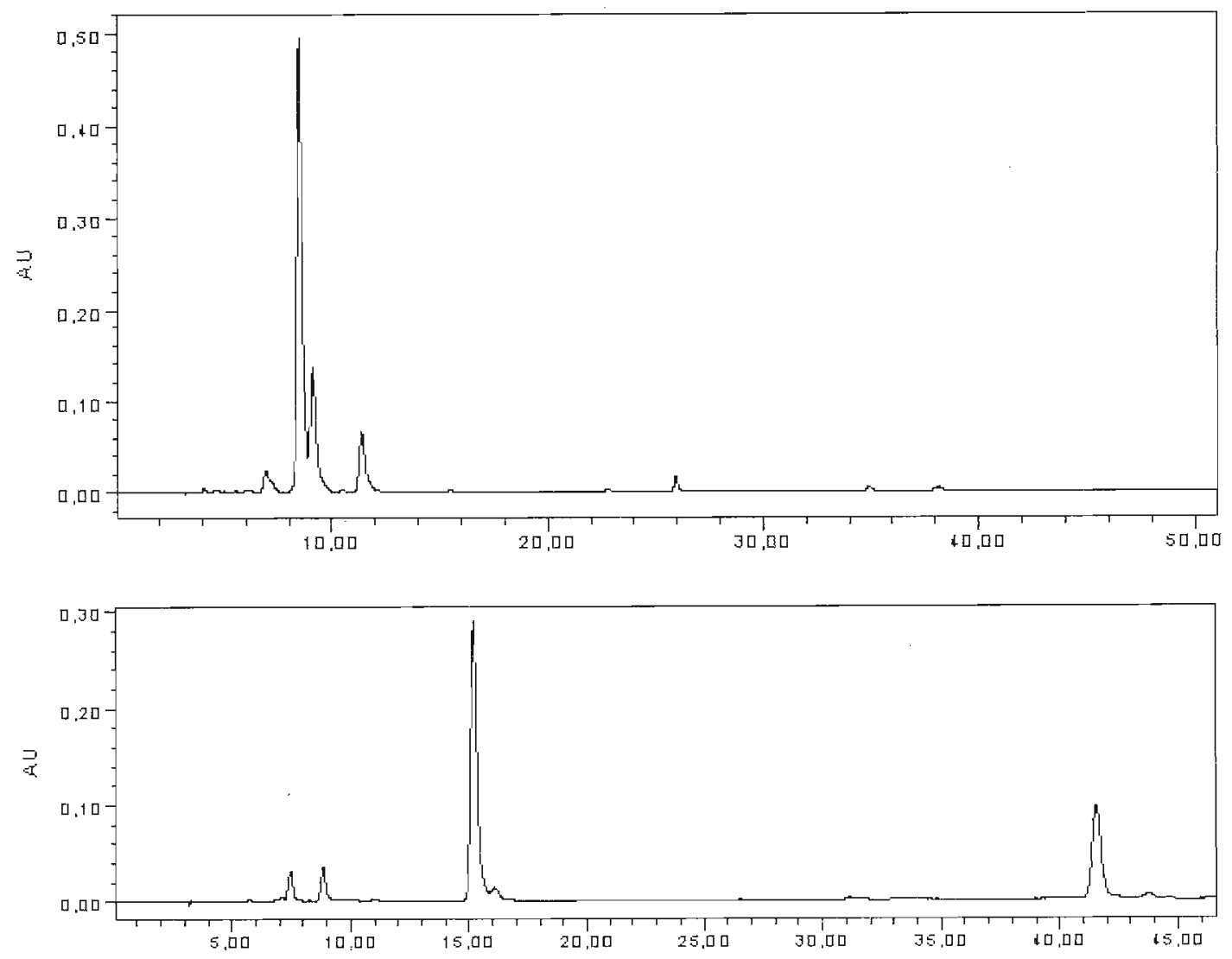

Figure 2: HPLC chromatogram of the (a) isolated standards (b) carotenoids of an extract of $l a v a l u$ (Chrysophyllum lanceolatum). Peak identifiction

1. neoxanthin 2. violaxanthin 3.cis-violaxanthin 4. $\beta$-cryptoxanthin monoepoxide

5. lutein 6. $\beta$-cryptoxanthin 7. -carotene 8. trans- $\beta$-carotene 


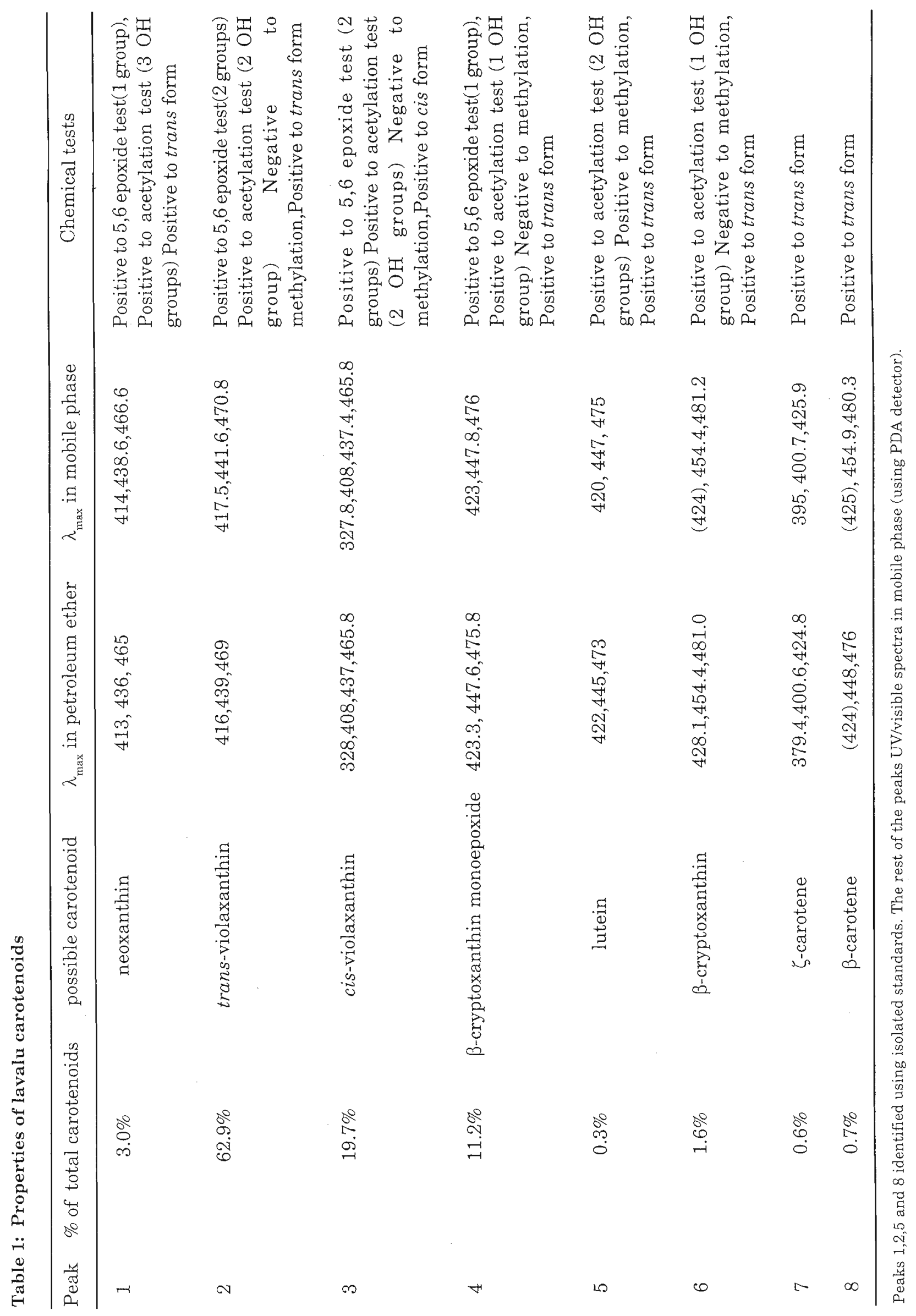


neoxanthin, violaxanthin, lutein and $\beta$-carotene was carried out using HPLC. Table 2 shows the contents of the carotenoids of lavalu.

The technique used in a previous study ${ }^{2}$ is cause for concern. It was felt that by not washing the carotenoids at the earliest, immediately after saponification could have caused artifacts. Further identification in that study was supported by only 3 standards (for 18 isolates). The nonidentification of the major compound violaxanthin in that study ${ }^{2}$ was surprising, as it should have eluted in the acetone: petroleum ether 20:80 fraction.

Table 2: Carotenoid concentrations in lavalu (Chysophyllum roxburghii)

\begin{tabular}{lc}
\hline Carotenoids & $\begin{array}{c}\text { Concentration } \\
\left(\mathrm{mgkg}^{-1} \text { wet weight }\right) \\
\text { Mean } \pm \mathrm{SD}\end{array}$ \\
\hline violaxanthine & $31.3 \pm 5.5$ \\
neoxanthin & $113.3 \pm 17.2$ \\
lutein & $0.4 \pm 0.1$ \\
$\beta$-carotene & $1.4 \pm 0.3$ \\
\hline
\end{tabular}

$n=4$

The present study shows that violaxanthin (a non pro-vitamin A carotenoid) dominates. This includes trans-violaxanthin $(62 \% \mathrm{w} / \mathrm{w})$ and cisviolaxanthin $(20 \% \mathrm{w} / \mathrm{w})$ of total carotenoids. Percentages given for individual carotenoids were from HPLC readings (Millennium 32 software) directly. Cis-violaxanthin was identified using cis peak at $328 \mathrm{~nm}$ of UV/visible spectrum and iodine catalised isomerisation reaction. All trans violaxanthin was obtained directly from the column as pure crystals. Since it is very polar and easily isolated pure, it could be used as standards in carotenoid analysis.
The percentage of $\beta$-carotene in $\mathrm{C}$. roxburghii $0.8 \%$ and other pro-vitamin $A$ carotenoids were $11 \%$. This represents very low retinol equivalent ( $68 \mathrm{RE} / 100 \mathrm{~g})$. As such it cannot be advocated as pro-vitamin A supplement as done in Phillipines (Rodeiguez-Amaya, personal communication). Further, the structural features of the carotenoids make bioconversion studies with animals or humans futile.

There is also a doubt that violaxanthin is not absorbed as such by humans ${ }^{4}$, and this may be the reason for there being no information regarding its health implications. Therefore antioxidant action of these fruit carotenoids is also questionable.

Perhaps the best use of the lavalu carotenoids is as a food colourant for oil based foods.

\section{References}

1. The Wealth of India (1952). A dictionary of Indian raw materials and Industrial products Vol. II Council of Scientific and Industrial Research, New Delhi.

2. Abeysekara M. (1993). Studies on betalaines in Beta vulgaris and carotenoid and carbohydrates in Chrysophyllum roxhburghii fruit. Ph. D Thesis University of Colombo, Sri Lanka.

3. Rodriguez-Amaya D. B. (1999), A Guide to Carotenoid Analysis in Foods. ILSI press, Washington.

4. Kimura M. \& Rodriguez-Amaya D. B. (2002). A scheme for obtaining standards and HPLC quantification of leafy vegetable carotenoids. Food Chemistry 78: 389-398.

5. Barua A. \& Olson J. A. (2001). Xanthophyll epoxides, unlike $\beta$-carotene monoepoxides, are not directly absorbed by humans. Journal of Nutrition 131: 3212-3215. 